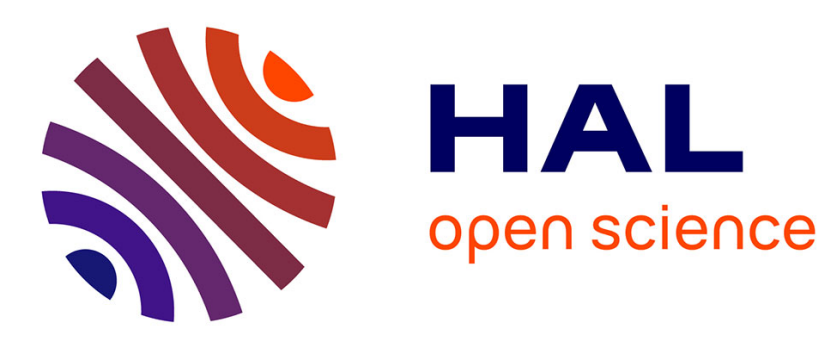

\title{
Metamodel-based Markov-Chain-Monte-Carlo parameter inversion applied in eddy current flaw characterization
}

Caifang Cai, Roberto Miorelli, Marc Lambert, Thomas Rodet, Dominique

Lesselier, Pierre-Emile Lhuillier

\section{To cite this version:}

Caifang Cai, Roberto Miorelli, Marc Lambert, Thomas Rodet, Dominique Lesselier, et al.. Metamodelbased Markov-Chain-Monte-Carlo parameter inversion applied in eddy current flaw characterization. NDT \& E International, 2018, 99, pp.13-22. 10.1016/j.ndteint.2018.02.004 hal-01634071

\section{HAL Id: hal-01634071 \\ https://hal-centralesupelec.archives-ouvertes.fr/hal-01634071}

Submitted on 3 Mar 2020

HAL is a multi-disciplinary open access archive for the deposit and dissemination of scientific research documents, whether they are published or not. The documents may come from teaching and research institutions in France or abroad, or from public or private research centers.
L'archive ouverte pluridisciplinaire HAL, est destinée au dépôt et à la diffusion de documents scientifiques de niveau recherche, publiés ou non, émanant des établissements d'enseignement et de recherche français ou étrangers, des laboratoires publics ou privés. 


\title{
Metamodel-based Markov-Chain-Monte-Carlo parameter inversion applied in eddy current flaw characterization
}

\author{
Caifang Cai ${ }^{\mathrm{a}}$, Roberto Miorelli, Marc Lambert ${ }^{\mathrm{c}}$, Thomas Rodet ${ }^{\mathrm{d}}$, Dominique Lesselier ${ }^{\mathrm{a}}$, \\ Pierre-Emile Lhuillier ${ }^{\mathrm{e}}$ \\ ${ }^{a}$ L2S, UMR CNRS 8506, CNRS-CentraleSupélec-Univ. Paris-Sud, Université Paris-Saclay, \\ 3 rue Joliot-Curie, 91192 Gif-sur-Yvette, France \\ ${ }^{b}$ Département Imagerie Simulation pour le Contrôle, CEA, LIST, 91191 Gif-sur-Yvette, France \\ ${ }^{c}$ GeePs, UMR CNRS 850\%, CentraleSupélec, Univ. Paris-Sud, Université Paris-Saclay, Sorbonne Universités, \\ UPMC Univ Paris 06, 3 \& 11 rue Joliot-Curie, 91192 Gif-sur-Yvette, France \\ ${ }^{d}$ SATIE, ENS-Cachan, Université Paris-Saclay, 61 avenue du Président Wilson, 94230 Cachan CEDEX, France \\ ${ }^{e}$ Departement MMC, EDF RED, EDF Lab Les Renardieres, 77818 Moret-sur-Loing, France
}

\begin{abstract}
Flaw characterization in eddy current testing usually requires to solve a non-linear inverse problem. Due to high computational cost, Markov Chain Monte Carlo (MCMC) methods are hardly employed since often needing many forward evaluations. However, they have good potential in dealing with complicated forward models and they do not reduce to only providing the parameters sought. Here, we introduce a computationally-cheap surrogate forward model into a MCMC algorithm for eddy current flaw characterization. Due to the use of a database trained off-line, we benefit from the MCMC algorithm for getting more information and we do not suffer from the computational burden. Numerous experiments are carried out to validate the approach. The results include not only the estimated parameters, but also standard deviations, marginal densities and correlation coefficients between two parameters of interest.
\end{abstract}

Keywords: Inversion, MCMC, eddy-current, metamodel, Bayesian.

\section{Introduction}

In eddy current flaw characterization, the aim is to extract information about defects possibly found within the inspected part. This information can usually be characterized by several parameters. As an example, a volume crack can be described by length, depth and opening width. Recovering them from observations is an inverse problem with a limited set of unknown parameters, here three. According to the state of art, analytical and statistical solution methods are proposed.

Analytical ones [4, 44] address the analytical relation between parameters and measurements and try to inverse it analytically. Yet, they are limited to special cases wherein this relation is simple enough so that analytical inversion is suitable. Furthermore, most are very sensitive to noise, and work only for high Signal-to-Noise Ratio (SNR) situations. Statistical ones are popular in parameter inversion because of high inversion accuracy and robustness vs. noise. Yet, what is of the most interest to us here is their ability of managing complicated models. As in the example above, flaw characterization in Eddy Current Testing (ECT) is an inverse problem usually involving a limited number of unknowns $[10,16]$. However, the problem is still difficult to solve due to the complexity of the forward model which describes the relation between flaw parameters and measurements, nonlinear in most situations.

Statistical methods are usually transformed into an optimization problem solved by a numerical optimization algorithm, as in $[1,14,16,37,47]$. Due to the complexity of the problem, statistical 
methods usually need to overcome two hurdles: high computational cost due to many evaluations of the forward model, non-convex objective function due to nonlinear parameter dependence. Gradient-based algorithms are widely used [1, 14, 16, 37, 47]. Yet, to calculate or approximate the gradient subject to the unknown parameters might not always be feasible. Furthermore, they cannot solve the non-convex problem, meaning to be blocked in a local minimum once in it.

Markov Chain Monte Carlo (MCMC) methods [24, 31, 45] have been used in ECT for Bayesian analysis $[19,36]$. However, they are not widely used for solving ECT inversion problems because of high computational cost. Though developments have been made in the last two decades to accelerate MCMC algorithms [12, 17, 23, 25], the computational burden remains heavy if willing to apply them directly in ECT.

We propose a surrogate model based MCMC approach to solve the ECT inverse problem. In this approach, a data-fitting surrogate forward model is introduced into a classical MCMC method where only interpolations are performed to approximate the forward evaluations during a MCMC process, then helping to reduce the computational cost. Since the employed MCMC algorithm is gradient-free and able to leave local minima, this makes it possible to solve non-convex problems. More importantly, by performing Bayesian analysis on MCMC results, it offers more information than the estimated parameters, like variances of estimates, correlation coefficients, and marginal posterior distributions. We describe the forward metamodel in $\S 2$, the MCMC inversion algorithm in $\S 3$, and we provide experimental validations in $\S 4$ and $\S 5$. An Appendix devoted to the forward solever and meatmodel generation follows.

\section{Data-fitting metamodel}

A general forward model with additive Gaussian noise can be described by

$$
\boldsymbol{y}=f(\boldsymbol{x})+\boldsymbol{\epsilon}, \boldsymbol{\epsilon} \sim \mathcal{N}\left(0, \sigma_{y}^{2}\right),
$$

where $\boldsymbol{x} \in \mathbb{R}^{N}, \boldsymbol{y} \in \mathbb{C}^{M}$ and $\boldsymbol{\epsilon}$ are unknown parameters, observations and noise, resp. $N$ is the number of unknown parameters while $M$ is the total number of measurement points. $\sigma_{y}^{2}$ is the noise variance of the same size as $\boldsymbol{y} . f(\boldsymbol{x})$ is the function that yields the physical relation between $\boldsymbol{x}$ and $\boldsymbol{y}$. Depending upon the configuration of inspection, different methods can be used to get $f(\boldsymbol{x})$. The Method of Moments (MoM) [9, 39] is one of the most used.

For statistical parameter inversion, thousands of forward evaluations could be required, hence, in effect overwhelming the algorithm if we employ MoM directly within the inversion. To overcome this problem, data-fitting surrogate models, also called metamodels [7, 21, 22, 30, 40], are proposed.

A metamodel includes a database trained off-line, independently from the inversion, and an on-line interpolator, called upon only when a forward evaluation is needed. Once the database is trained, it can be applied onto all problems which are involving the same forward modeling, helping to significantly save cost whether repetitive use.

In our situation, the database is adaptively trained using the CIVA software where a MoM simulator $[32,33]$ is employed in order to perform the forward evaluation during the said training. Denoting $\mathcal{D}=\left\{\left\{\boldsymbol{x}_{j}, \bar{f}\left(\boldsymbol{x}_{j}\right)\right\}, j=1,2, \cdots, J\right\}$ as the metamodel database, $\bar{f}\left(\boldsymbol{x}_{j}\right)$ is the MoM simulator and $J$ is the total number of database pairs. Details on this adaptive database training are found in $[7,21]$. In brief, a global approximation is achieved, $\left|\frac{f\left(\boldsymbol{x}_{j}\right)-\bar{f}\left(\boldsymbol{x}_{j}\right)}{\bar{f}\left(\boldsymbol{x}_{j}\right)}\right| \leq \epsilon$ for all $\boldsymbol{x}_{j} \in \mathcal{D}$, by sequentially refining the parameter space where $\epsilon$ is the relative tolerance for the adaptive database training, set to $10^{-2}$ in our situation.

For a given problem, $J$ depends upon the smoothness of $\bar{f}(\boldsymbol{x})$. Fortunately, $\bar{f}(\boldsymbol{x})$ is a smooth function in most ECT applications due to the use of low frequencies and the high conductivity 
of the media under inspection. Thus, the global approximation accuracy required in the adaptive database training can be achieved with a limit number of pairs.

As for the interpolation, multi-linear [5], kriging [8] or sparse-grid [6] methods have been proposed in our previous work. Multi-linear interpolation is fast in terms of computation for a small number of unknowns, kriging is more accurate but requires the pre-estimation of the covariance matrix while sparse-grid is more suitable to high dimensional problems. In terms of time spared compared with a MoM simulator, it can be several orders of magnitude. Let us take the two examples discussed in $\S 4$ and $\S 5$, a MoM simulator needs seconds to perform one forward simulation while a multi-linear interpolation only needs milliseconds (on a $3.4 \mathrm{GHz} \mathrm{PC}$ ). In the inversion, the time saving will be multiplied by the number of iterations, which is often more than thousands for a MCMC method.

\section{MCMC parameter inversion}

\subsection{Posterior mean}

Least Square (LS) and Weighted Least Square (WLS) are the most used inversion criteria in ECT. For well-posed problems, LS solution yields a good estimator for $\boldsymbol{x}$. Yet ECT is often ill-posed, and for noisy data and problems with many unknown parameters, multiple solutions might exist. To handle multi-solution and local minima, we propose the Posterior Mean (PM) inversion approach $[11,27]$

$$
\hat{\boldsymbol{x}}=E\left[p\left(\boldsymbol{x} \mid \boldsymbol{y}, \sigma_{y}^{2}, \boldsymbol{\theta}\right)\right]=E\left[p\left(\boldsymbol{y} \mid \boldsymbol{x}, \sigma_{y}^{2}\right) p(\boldsymbol{x} \mid \boldsymbol{\theta})\right]
$$

to be tackled by a MCMC simulation algorithm. In Eq. (2), $p\left(\boldsymbol{x} \mid \boldsymbol{y}, \sigma_{y}^{2}, \boldsymbol{\theta}\right), p\left(\boldsymbol{y} \mid \boldsymbol{x}, \sigma_{y}^{2}\right)$ and $p(\boldsymbol{x} \mid \boldsymbol{\theta})$ respectively denote posterior probability, likelihood, and prior. $\boldsymbol{\theta}$ is the hyper-parameter associated with the prior model. For the uniform one here (given in Eq. (3)), it corresponds to the parameter ranges.

The likelihood can be obtained from the Gaussian noise model in Eq. (1). The prior $p(\boldsymbol{x} \mid \boldsymbol{\theta})$ corresponds with the information known about $\boldsymbol{x}$. E.g., if $\boldsymbol{x}$ represents the dimension parameters of a 3-D volume flaw, all non-negative and within a certain range. Thus, an uniform prior model can be used:

$$
p(\boldsymbol{x} \mid \mathcal{M})=\frac{\chi_{\mathcal{U}}(\boldsymbol{x})}{\operatorname{volume}(\mathcal{U})}, \mathcal{U}=\left[a_{1}, b_{1}\right] \times \cdots \times\left[a_{N}, b_{N}\right]
$$

where $\mathcal{U}$ is the uniform parameter space with $N$ denoting the number of unknown parameters. $\chi_{\mathcal{U}}(\boldsymbol{x})$ is the indicator function on $\mathcal{U}$. All values of our interest for the $i$-th parameter $x_{i}$ in the vector $\boldsymbol{x}$ should be included in the corresponding range between $a_{i}$ and $b_{i}$.

This uniform model is a general choice without further prior information. A different prior model can be employed according to available information for parameters of interest, e.g. a lognormal distribution can be used for lift-off. However, this will not change too much the inversion result when the measurements are informative enough, which is the case for the two examples discussed in $\S 4$ and $\S 5$.

\section{2. $M C M C$ sampling}

From Eq. (2), we see that PM estimation corresponds with the expectation of the posterior distribution. Due to the multi-dimensionality and the complexity of the posterior distribution, it is still hard to calculate its expectation even with a given Gaussian likelihood and an uniform prior. So, we follow an alternative path, a MCMC simulation method, to approximate this value.

MCMC is a family of numerical sampling methods enabling to generate samples following a given distribution. We use the algorithm Random Walk Metropolis-within-Gibbs (RW-MWG) proposed by Tierney [45] based on the classical Metropolis-Hasting method [29, 31]. The author 
adopted Gibbs sampler in the Metropolis-Hasting method in order to accelerate the sampling for independent prior distributions. Fig. 1 illustrates its implementation in our situation. The forward evaluation $f(\boldsymbol{x})$ is called in the decision of acceptance, where it corresponds to an interpolation based on the pre-trained database instead of a MoM simulator $\bar{f}(\boldsymbol{x})$.

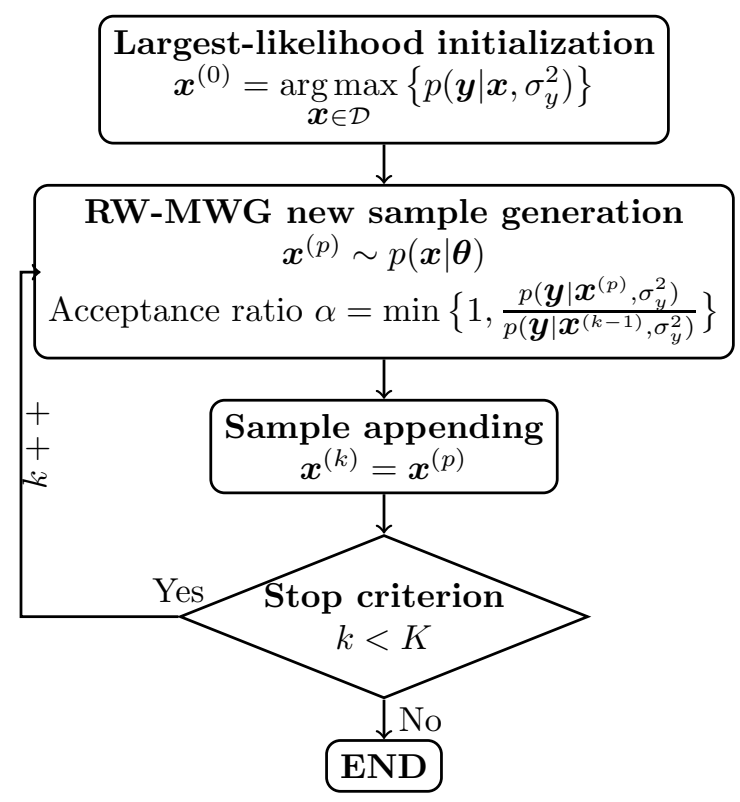

Figure 1: Implementation of metamodel-based MCMC algorithm for inversion.

The main discrepancy between our implementation and the classical RW-MWG method is the initialization. Instead of a random sample, we use the sample corresponding with the largest likelihood value among all samples within the metamodel database as our initialization. As illustrated in Fig. 2, this can help to reduce the warm-up iterations, which are time-consuming and introduce a bias on the parameter estimation. When the posterior is distributed only on a small part of the entire prior volume, problems of warm-ups are also more crucial. Unfortunately, most of the problems which we are dealing with here are as such. Since the sample with largest likelihood is never too far away from the true parameters, a much smaller number of warm-ups will be needed when we simply initialize from this sample.

\subsection{Results from a MCMC algorithm}

Compared with other parameter inversion algorithms, like LS and Maximum Likelihood (ML) (with help of numerical optimization), an advantage of the MCMC algorithm is to provide more than just flaw parameters.

\section{- Parameter estimation}

Upon running a MCMC algorithm, a series of samples $\left\{\boldsymbol{x}^{(1)}, \boldsymbol{x}^{(2)}, \cdots, \boldsymbol{x}^{(K)}\right\}$ is available, $K$ is the total number of iterations in Fig. 1. Since they follow the posterior distribution $p(\boldsymbol{x} \mid \boldsymbol{y})$, we get parameter estimates. PM estimators in Eq. (2) can be approximated by averaging:

$$
\hat{x}_{i} \approx \frac{1}{K} \sum_{k=1}^{K} x_{i}^{(k)}, \quad i=1,2, \cdots, N .
$$

\section{- Parameter estimation variance}

Estimation variances follow at no additional cost:

$$
\sigma_{i}^{2}=\frac{1}{K(K-1)} \sum_{k=1}^{K}\left(x_{i}^{(k)}-\hat{x}_{i}\right)^{2}, \quad i=1,2, \cdots, N,
$$




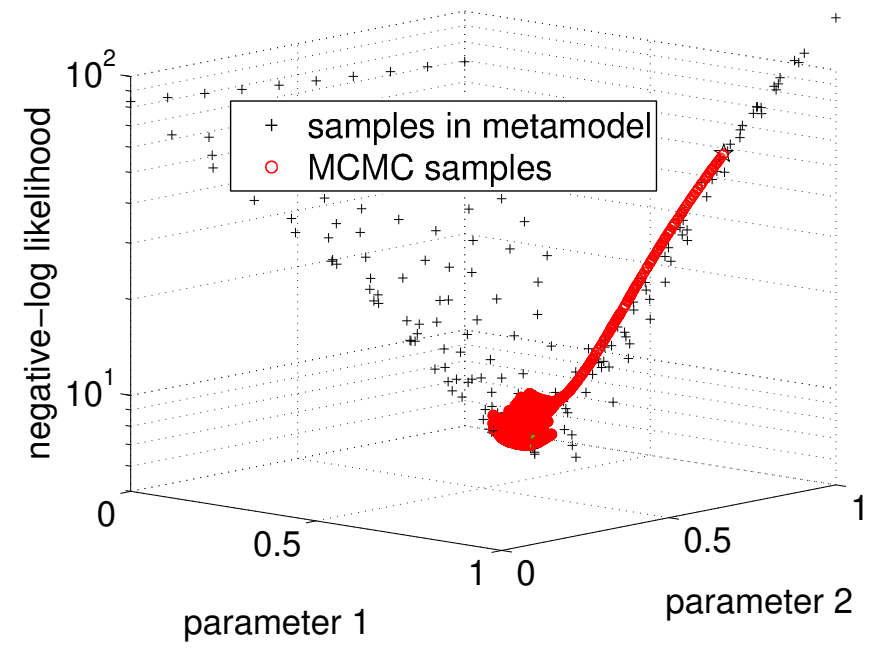

(a) random initialization far away from true parameters, long tail of warm-ups

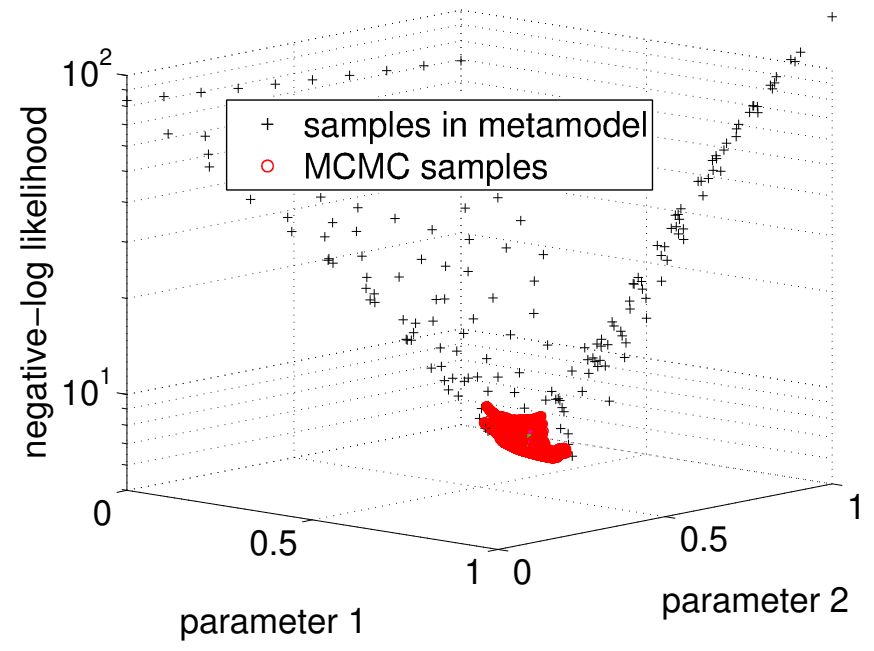

(b) largest-likelihood initialization

Figure 2: Comparison between random and largest-likelihood initialization; the true parameters are located within the concave bottom of the negative-log likelihood mesh.

quantifying estimation uncertainty, $\hat{x}_{i}$ estimated parameter in Eq. (4).

\section{- Marginal posterior distributions}

From MCMC samples, we get the posterior distribution by calculating their histograms. However, for a large number of unknowns $(N>3)$, it is difficult to visualize this distribution. We are more keen to marginal distributions along each parameter (1D marginal) or within planes of two parameters (2D marginal). Taking 1D distributions as examples, the latter are approximated by

$$
p\left(x_{i} \mid \boldsymbol{y}\right) \approx \operatorname{histogram}\left(\left\{x_{i}^{(1)}, x_{i}^{(2)}, \cdots, x_{i}^{(K)}\right\}\right), i=1, \cdots, N .
$$

For 2D ones, we calculate the 2D histograms on the MCMC samples.

\section{- Correlation coefficient}

Correlation coefficient $\rho\left(x_{i}, x_{j}\right)$ between two parameters $x_{i}$ and $x_{j}$ follows as well, helping to understand the difficulty in parameter inversion and relative bias if extra noise:

$$
\rho\left(x_{i}, x_{j}\right)=\frac{\sum_{k=1}^{K}\left(x_{i}^{(k)}-\bar{x}_{i}\right)\left(x_{j}^{(k)}-\hat{x}_{j}\right)}{\sigma_{i} \sigma_{j}}, i \neq j ; i, j=1, \cdots, N .
$$

$\hat{x}_{i}, \hat{x}_{i}$ are the estimated parameters in Eq. $4 ; \sigma_{i}, \sigma_{j}$ are the corresponding standard deviations obtained in Eq. (5). $\rho\left(x_{i}, x_{j}\right)$ is between -1 and 1 . The closer to zero, the less correlation between $x_{i}$ and $x_{j}$. Strong correlation indicates that the parameters are difficult to estimate. A small misestimate of one can lead to a large misestimate of the other, and weak noise can cause large errors on both. Yet, low correlation does not mean easy estimation, since this depends on the form of the posterior distribution. The topic will be discussed later in $\S 4$ and $\S 5$.

\subsection{Discussion on $M C M C$ parameter inversion}

The sampling number $K$ needs to ensure the convergence of the MCMC process. [18] presents different ways of determining the MCMC convergence. However, most of them are post-analysis 
Table 1: WFNDE 2008 eddy current benchmarks.

\begin{tabular}{cccc}
\hline \hline Flaw & \multicolumn{3}{c}{ flaw parameters } \\
& depth $d$ & length $l$ & width $w$ \\
\hline $40 \mathrm{I}$ & 0.62 (from top) & 20 & 0.11 \\
$80 \mathrm{I}$ & 1.24 (from top) & 20 & 0.14 \\
$40 \mathrm{E}$ & 0.62 (from back) & 20 & 0.11 \\
$80 \mathrm{E}$ & 1.24 (from back) & 20 & 0.14 \\
\hline
\end{tabular}

methods. We follow a more practical strategy where $K$ is chosen to be of sufficient large value, guaranteeing the convergence according to experience, e.g., $K=10^{4}$ for the 3 -parameter case in $\S 4, K=10^{5}$ for the 5 -parameter case in $\S 5$.

The RW-MWG method used in this work is a classical MCMC algorithm, it is gradient-free and it can be applied to all kinds of ECT inversion problems regardless of the forward complexity and the chosen prior model. One of its shortcomings is that the required sampling number $K$ ensuring the convergence of the MCMC process increases rapidly with the number of unknown parameters. Thus, it is not suitable for solving large-dimensional problems.

Recent works in MCMC have led to many advanced algorithms, like adaptive MCMC [3, 26], particle MCMC [2, 20] and hybrid MCMC [35, 38]. With proper extension, these algorithms can also be employed here to accelerate the algorithm. However, these improvements in MCMC often require further information. For example, the adaptive MCMC requires to estimate the covariance matrix of the unknowns; hybrid MCMC needs to refer to gradient or even Hessian information of the objective distribution. In our case, such information is not always easy to obtain. This is also the reason why we propose to use a classical MCMC method, RW-MWG discussed in $\S 3.2$.

\section{Benchmark experimental validation}

\subsection{Benchmark set-up \& measurements}

To validate our metamodel-based MCMC parameter inversion, we first apply it to slab inspection in the 2008 ECT benchmark of World Federation of Nondestructive Evaluation (WFNDE) centers [43], sketched in Fig. 3. We aim at retrieving flaw dimensions from surface scans of impedance variations.

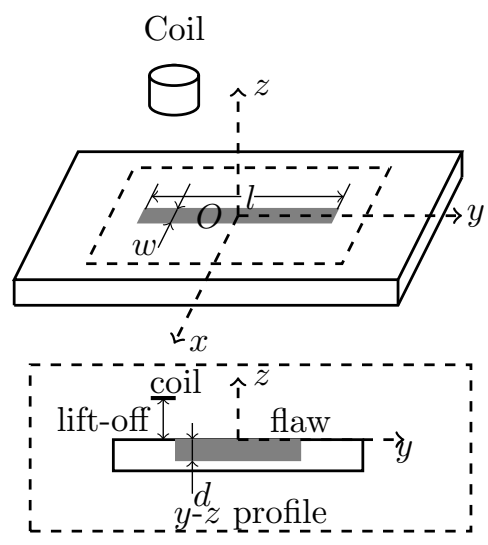

Inspected inconel plate

thickness: $1.55 \mathrm{~mm}$

conductivity: $10^{6} \mathrm{~S} / \mathrm{m}$

relative permeability: 1

\section{Inspection}

frequency: $300 \mathrm{kHz}$

lift-off: $0.303 \mathrm{~mm}$

steps: $0.5 \times 0.5 \mathrm{~mm}^{2}$

internal diameter: $2 \mathrm{~mm}$

number of steps: $41 \times 57$

external diameter: $3.25 \mathrm{~mm}$

height: $2 \mathrm{~mm}$

turns: 328

Figure 3: Sketch of slab flaw inspection problem in 2008 WFNDE eddy current benchmark.

Four different flaws are considered, two open at the top surface of the slab, two at its bottom surface, referred as 40I, 80I, 40E and 80E, resp. Tab. 1 provides depths $d$, lengths $l$, and widths $w$. 
Fig. 4 displays maps of amplitudes. Those for flaws opening at the bottom surface are generally smaller than for those opening at the top surface. And the one for $40 \mathrm{E}$ is so small that SNR is only about $9 \mathrm{~dB}$. So, one question is whether the approach correctly yields the flaw parameters.

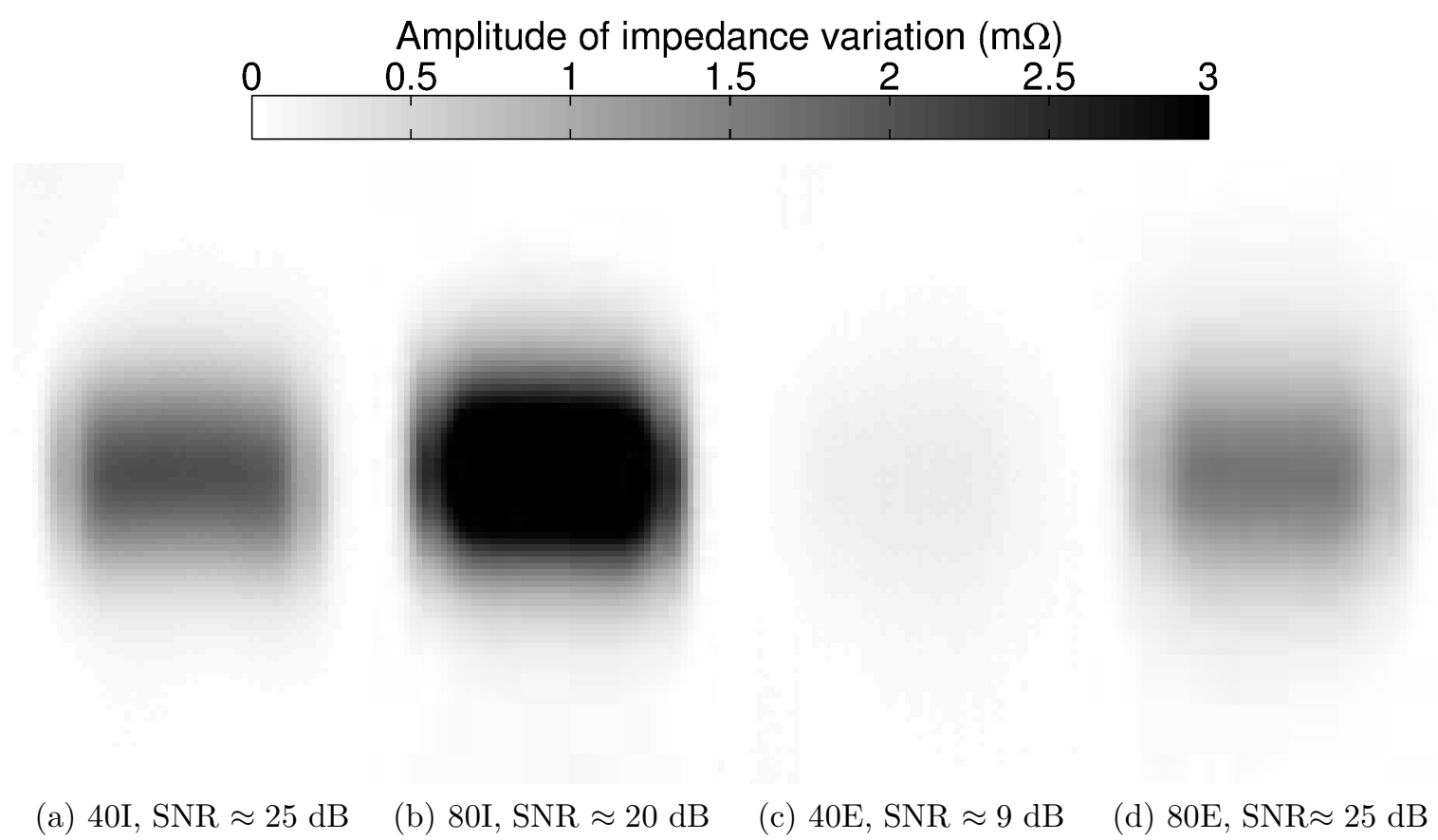

Figure 4: Amplitude maps of measurements and approximated SNRs.

\subsection{Metamodel database training}

For the benchmark test case, we first trained two databases, one for top-surface flaws, the other for back-surface flaws. Three flaw characteristics, depth, length and width, are taken as unknown. An adaptive training procedure [21] based on a multi-linear interpolator [5] is employed. Therein, we initialize with a certain number of random points, samples being added iteratively at positions where interpolation uncertainty is large. The process stops when maximal uncertainty is smaller than a threshold. For all input points, outputs are simulated by MoM [33] in aforementioned CIVA.

Table 2: WFNDE2008 tests - metamodel parameter bounds and total number of samples.

\begin{tabular}{cccccc|c}
\hline \multicolumn{5}{c|}{ Parameter bounds } & \multirow{2}{*}{ number } \\
$d(\%$ plate thickness $)$ & $l(\mathrm{~mm})$ & $w(\mathrm{~mm})$ & of points \\
inf. & sup. & inf. & sup. & inf. & sup. & \\
\hline 25 & 85 & 13 & 23 & 0.08 & 0.22 & 2000 \\
\hline
\end{tabular}

Tab. 2 summarizes these two metamodels (identical for top-surface flaw model and backsurface flaw model). The bounds for each parameter are chosen according to the range of flaw dimensions of our interest. In terms of computational cost, there are 2000 input-output pairs in each metamodel. So we need to run 2000 simulations by using the method in [33]. However, those can be performed off-line. Once the database available, it can be used for all measurements with the same modeling. The bounds on the parameters can also be extended in order to include more flaw dimensions. 
Table 3: Estimated flaw parameters for WFNDE 2008 benchmark measurements, relative errors larger than 10\% in bold.

\begin{tabular}{cccc}
\hline \hline Flaw & \multicolumn{3}{c}{ estimated parameters $\hat{\boldsymbol{x}} \pm \boldsymbol{\sigma}(\mathrm{mm})$} \\
& $d$ & $l$ & $w$ \\
\hline \multirow{2}{*}{$40 \mathrm{I}$} & 0.62 & 20 & 0.11 \\
& $\mathbf{0 . 4 8 8} \pm 0.001$ & $19.828 \pm 0.022$ & $\mathbf{0 . 2 2 5} \pm 0.002$ \\
\hline \multirow{2}{*}{$80 \mathrm{I}$} & 1.24 & 20 & 0.14 \\
& $1.129 \pm 0.001$ & $19.937 \pm 0.007$ & $\mathbf{0 . 2 1 9} \pm 0.001$ \\
\hline \multirow{2}{*}{$40 \mathrm{E}$} & 0.62 & 20 & 0.11 \\
& $0.607 \pm 0.003$ & $19.231 \pm 0.097$ & $\mathbf{0 . 2 4 4} \pm 0.007$ \\
\hline \multirow{2}{*}{$80 \mathrm{E}$} & 1.24 & 20 & 0.14 \\
& $1.228 \pm 0.001$ & $19.924 \pm 0.008$ & $0.156 \pm 0.002$ \\
\hline
\end{tabular}

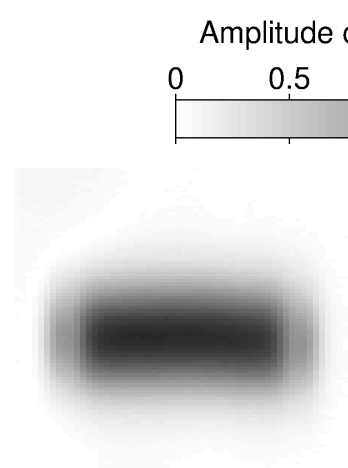

(a) experimental

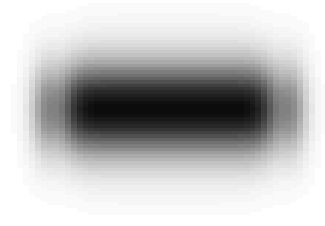

(b) simulated at estimated parameters reference parameters

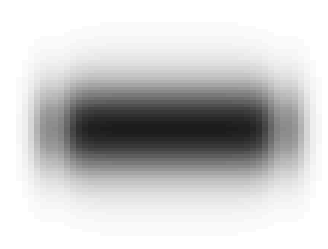

(c) simulated at
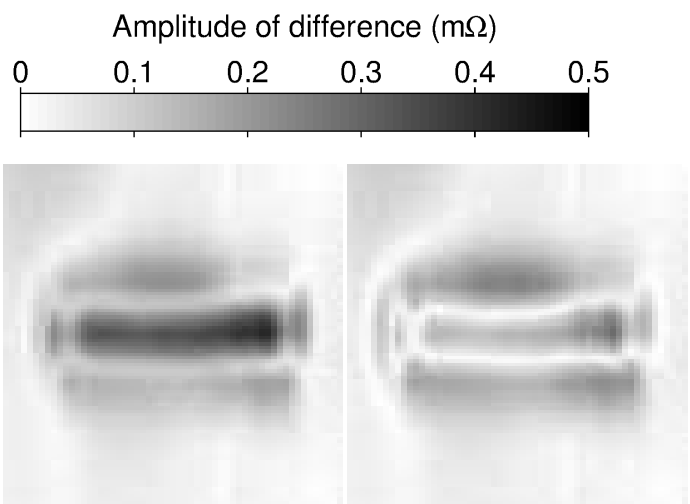

(d) $|(\mathrm{b})-(\mathrm{a})|$, RMSRE $=2.56 \%$ (e) $|(c)-(a)|$, $\mathrm{RMSRE}=1.42 \%$

* RMSRE: Root Mean Squared Relative Error.

Figure 5: Amplitudes of simulated noise-free data at estimated flaw parameters $(\hat{l}, \hat{w}, \hat{d})=(0.49,19.83,0.23)$ mm and at reference flaw parameters $(l, w, d)=(0.62,20,0.11) \mathrm{mm}$ in comparison with experimental measurements for flaw 40I in WFNDE 2008 benchmarks.

\subsection{MCMC parameter estimation results}

By applying the metamodel-based MCMC algorithm, we obtain the results displayed in Tab. 3, reference parameters being in gray. Flaw lengths are very well estimated with relative errors less than $1 \%$ whereas depths and widths are not, especially widths. Except for 80E, the estimated ones are close to $0.25 \mathrm{~mm}$ while reference values are $0.11 \mathrm{~mm}$ and $0.14 \mathrm{~mm}$. Such a small flaw width appears out of the inversion ability based on current inspection configuration. The measurement error is larger than the difference introduced between flaws with widths of $0.11 \mathrm{~mm}$ and $0.25 \mathrm{~mm}$. To illustrate the topic further, we give in Fig. 5 the simulated noise-free impedance variations at estimated and reference sizes compared with experimental measurements for the worst case flaw, 40I. From the differences of amplitudes in Figs. 5d and 5e, simulated data at estimated parameters appear closer to the experimental ones than the simulated data at reference parameters.

As discussed in $\S 3.3$, the MCMC algorithm more than estimated parameters and variances. We show in Fig. 6 the evolution of MCMC samples vs. iterations for the best case of parameter inversion, flaw 80E. The algorithm converges fast, after a short period of warm-up (around 400 iterations). Compared with the total number of iterations $\left(K=10^{4}\right)$, warm-up iterations are few. This is due to the use of the largest-likelihood initialization. We imagine that it could be far away from the true value if we were to randomly initialize, so many warm-up iterations would be necessary. In consequence, the parameter inversion results should be more biased because of those. 

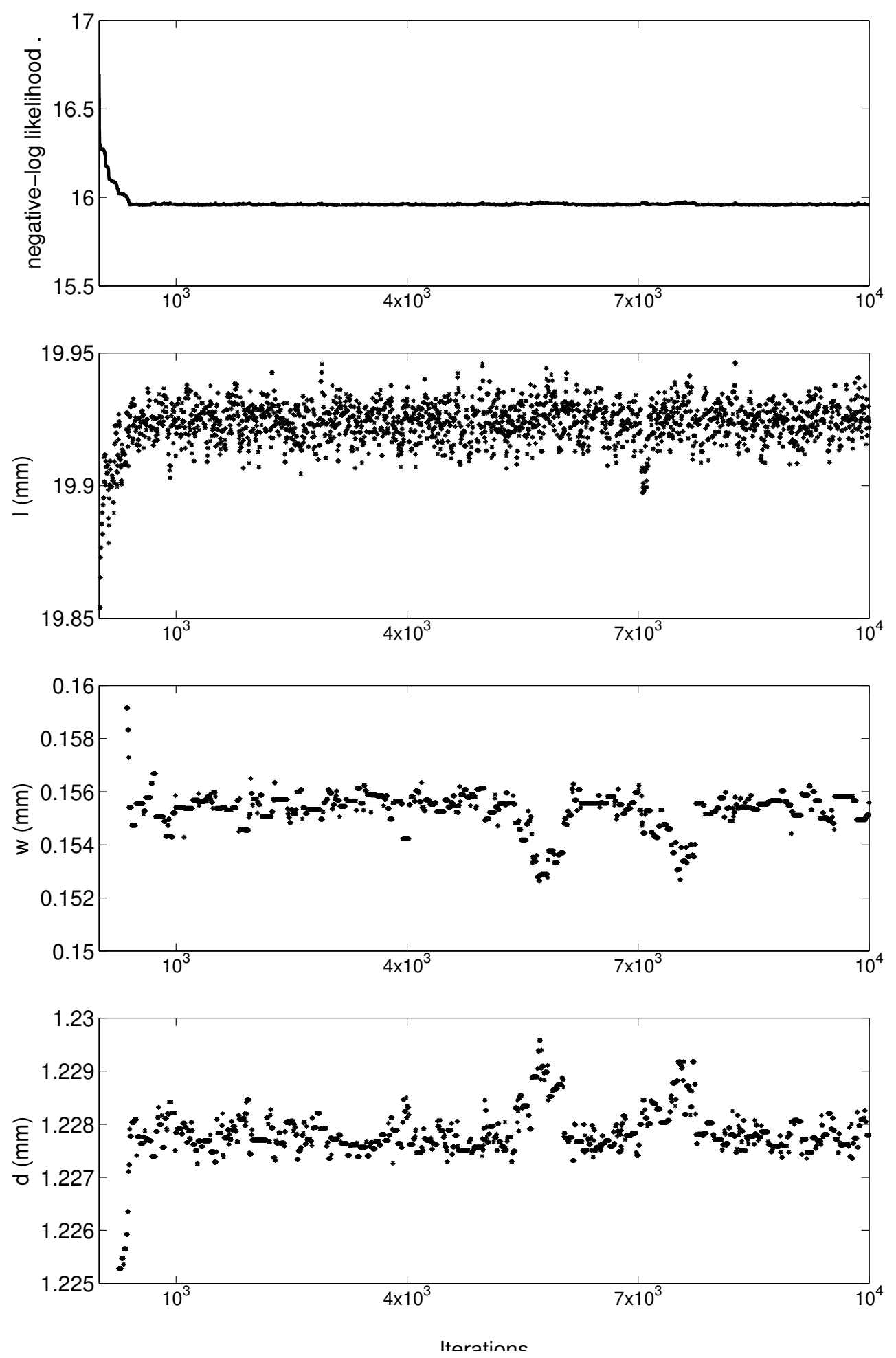

Figure 6: MCMC samples versus iterations for flaw 80E.

From Fig. 6, we also see that the likelihood remains nearly the same after warming-up. If a square error based criterion is used for inversion, the algorithm could be stopped at any of the values after warm-ups. In contrast, the proposed PM inversion is always able to provide an unique solution even for complicated problems where the likelihood distribution has a flat top or when multiple least-square solutions exist.

From Fig. 7, we see that estimating the sizes of a single flaw is simple, and the estimated 1D marginal distributions are close to Gaussian ones. We also see that flaw depth and width are 

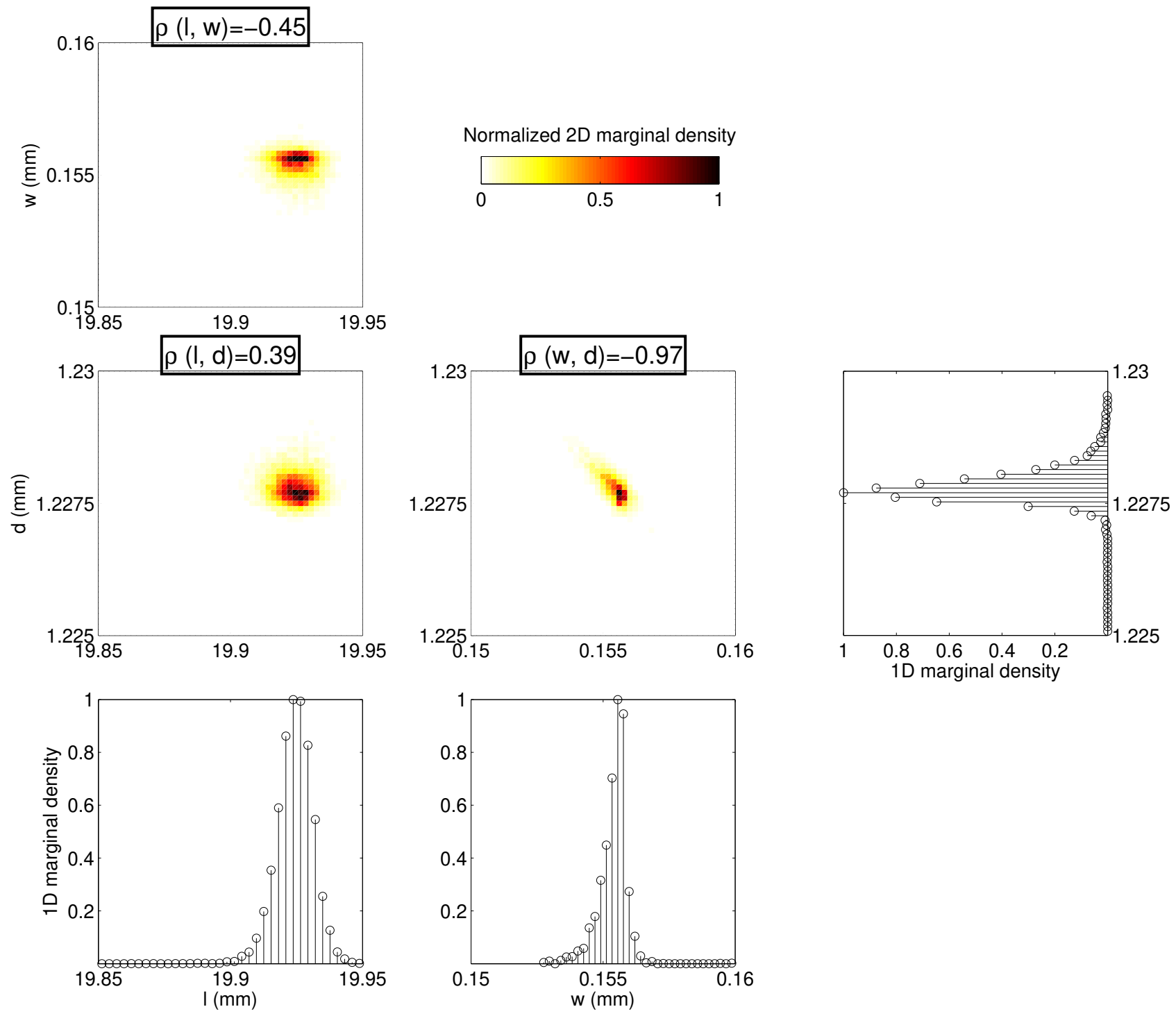

Figure 7: 2D distribution of MCMC samples (1st two rows) and 1D marginal distributions (last row) estimated from histograms of MCMC samples for flaw $80 \mathrm{E}$, correlation coefficients in boxed text on top of $2 \mathrm{D}$ sample distribution maps. 
highly correlated, with $\rho(w, d)=-0.97$.

\section{Experimental validation in industry context}

In $\S 4$, we applied the metamodel-based MCMC inversion method on benchmarks. The results showed sufficient accuracy for practical use. But nuisance parameters, like lift-off, plate thickness, material homogeneity, cannot be exactly known. Next, we show how it works in such situations.

\subsection{Industry test example}

The test data are of a surface scan with an array probe [34] on an Inconel 600 plate of thickness $1.27 \mathrm{~mm}$ (standard thickness for steam generator tube in nuclear power plants). Several flaws of different sizes are present at the top surface, see Fig. 8. Because of fabrication and manual inspection, lift-off and plate thickness are known only approximately. In the forward modeling, a single-flaw model is used, so measurements corresponding to the flaw of interest are extracted from the whole scan.

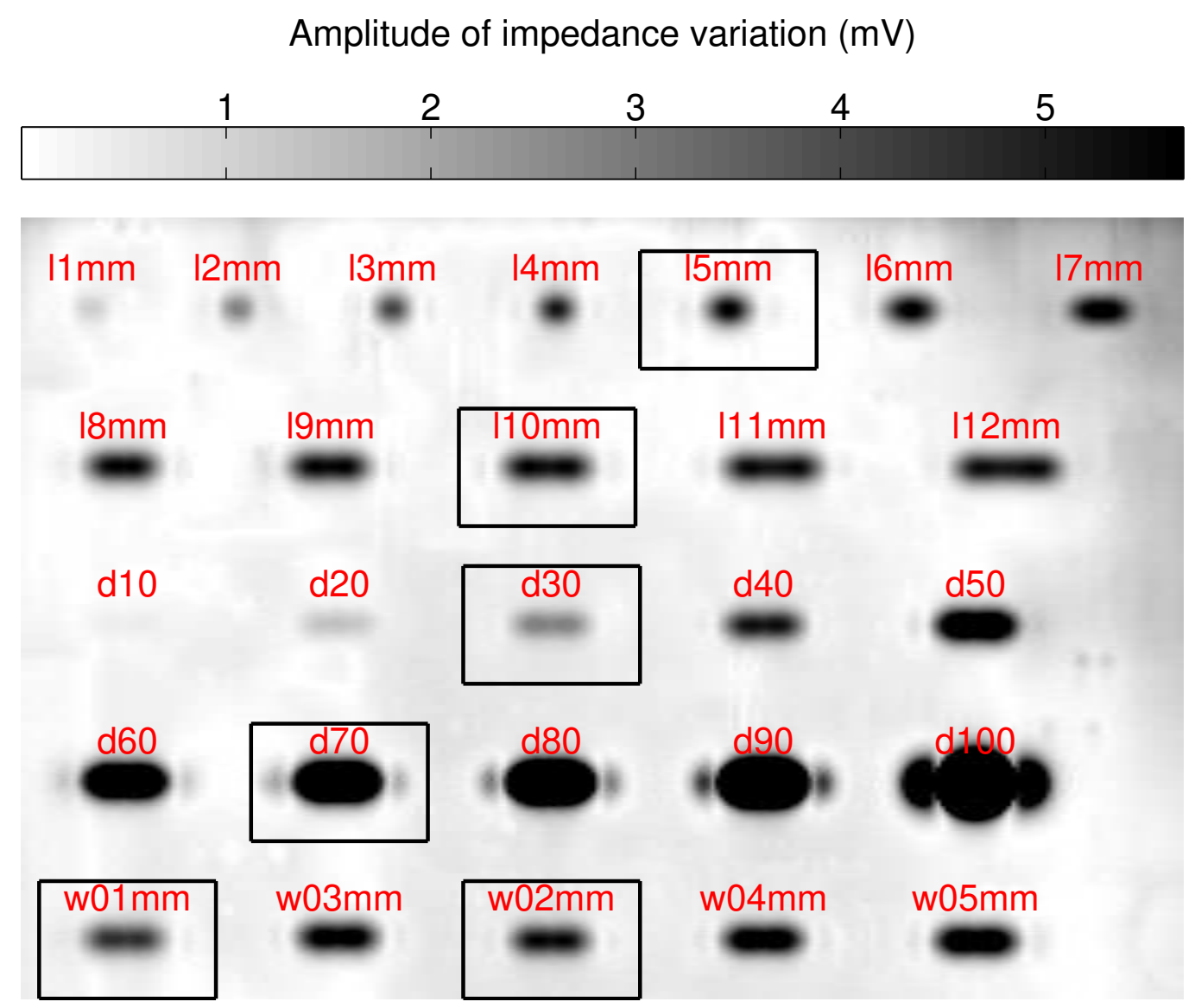

Figure 8: Measurements with an array probe, only flaws $15 \mathrm{~mm}, 110 \mathrm{~mm}, w 01 \mathrm{~mm}, w 02 \mathrm{~mm}, d 30$ and $d 70$ (in box) are discussed.

To avoid mis-quantification of flaw sizes from uncertain lift-off and plate thickness, we take them as unknown in the characterization. As in the above, we first train a database for a singleflaw model where flaw dimensions (depth, length, width), lift-off and plate thickness are unknown parameters. Bounds are chosen from prior information. Then, we apply the metamodel-based MCMC inversion approach in $\S 3$ individually upon extracted measurements with only one flaw within the field of view. 
Table 4: Estimated parameter results using $10^{5}$ MCMC samples from array probe experimental data. Reference values are in gray, results with bias larger than $15 \%$ in bold.

\begin{tabular}{cccccc}
\hline \hline \multirow{2}{*}{ Flaw } & \multicolumn{5}{c}{ estimated parameters $\hat{\boldsymbol{x}} \pm \boldsymbol{\sigma}_{e}(\mathrm{~mm})$} \\
& $l_{o}$ & $l$ & $w$ & $d$ & $h$ \\
\hline \multirow{2}{*}{$l 5 \mathrm{~mm}$} & 1 & 5 & 0.2 & 0.508 & 1.27 \\
& $\mathbf{0 . 8 9} \pm 0.10$ & $4.91 \pm 0.24$ & $0.17 \pm 0.12$ & $\mathbf{0 . 4 2 5} \pm 0.063$ & $1.21 \pm 0.03$ \\
\multirow{2}{*}{ l10mm } & 1 & 10 & 0.2 & 0.508 & 1.27 \\
& $1.00 \pm 0.13$ & $10.06 \pm 0.27$ & $\mathbf{0 . 2 4} \pm 0.09$ & $0.484 \pm 0.074$ & $1.27 \pm 0.03$ \\
$w 01 \mathrm{~mm}$ & 1 & 9 & 0.1 & 0.508 & 1.27 \\
& $0.98 \pm 0.16$ & $9.04 \pm 0.32$ & $\mathbf{0 . 2 7} \pm 0.09$ & $0.449 \pm 0.087$ & $1.28 \pm 0.03$ \\
$w 02 m m$ & 1 & 9 & 0.2 & 0.508 & 1.27 \\
& $1.00 \pm 0.15$ & $9.03 \pm 0.37$ & $0.22 \pm 0.09$ & $0.499 \pm 0.081$ & $1.28 \pm 0.04$ \\
d30 & 1 & 9 & 0.2 & 0.381 & 1.27 \\
& $1.08 \pm 0.21$ & $9.12 \pm 0.32$ & $0.22 \pm 0.10$ & $0.365 \pm 0.073$ & $1.26 \pm 0.05$ \\
d70 & 1 & 9 & 0.2 & 0.889 & 1.27 \\
& $0.95 \pm 0.05$ & $9.20 \pm 0.11$ & $\mathbf{0 . 2 9} \pm 0.04$ & $0.788 \pm 0.031$ & $1.21 \pm 0.02$ \\
\hline
\end{tabular}

\section{2. $M C M C$ results}

We discuss six typical flaws: $15 \mathrm{~mm}, 110 \mathrm{~mm}, w 01 \mathrm{~mm}, w 02 \mathrm{~mm}$, d30 and $d 70$, with reference sizes in Tab. 4 in gray. Inversion results with corresponding bias larger than $10 \%$ are in bold. The flaw width appears still hard to estimate because of small value and high correlation with others. Furthermore, for most cases, $w$ is over-estimated, $d$ under-estimated, confirming the inverse correlation relation in $\S 4$.

For an inversion with five unknowns, it is not easy to exhibit the posterior distribution. We display in Fig. 9 the 2D and 1D marginal distributions for flaw $110 \mathrm{~mm}$. The posterior distribution is more complicated than Gaussian. The correlation coefficient $\rho\left(x_{i}, x_{j}\right)$ cannot fully describe the correlation, e.g., between $w$ and $l_{o}$, we have complicated correlation from the $2 \mathrm{D}$ marginal distribution yet $\rho\left(w, l_{o}\right)$ is -0.09 .

Due to $l_{o}$ and $h$ being unknown parameters, this 5 -parameter inversion is far more difficult to solve than the 3-parameter one in $\S 4$. From the $2 \mathrm{D}$ marginal distributions, we observe that multiple local or even global maxima occur (multiple peaks on 2D marginal distributions in Fig. 9). So a gradient-based inversion could be blocked in any of these local maxima. This is also one main interest of the MCMC-based inversion approach, to manage problems with local minima and yield marginal distributions in addition.

\section{Conclusions}

We have presented a general framework of metamodel-based MCMC parameter inversion. Due to a pre-trained database, a MCMC method becomes usable in terms of computational cost. Thousands of forward evaluations are replaced by cheap interpolations. We have discussed how a classical MCMC method can be applied to flaw characterization in eddy current testing. We have shown that variances of the estimation, correlation coefficients and marginal posterior distributions can be obtained from MCMC results also. The approach has been tested on experimental data: one with flaw sizes as unknown parameters, one with lift-off and plate thickness unknown as well. The analysis has been run on eddy-current cases, but applies to other testings, ultrasound, infrared, radio-frequency, etc. The main limit is that the number of unknown parameters be not too large. 


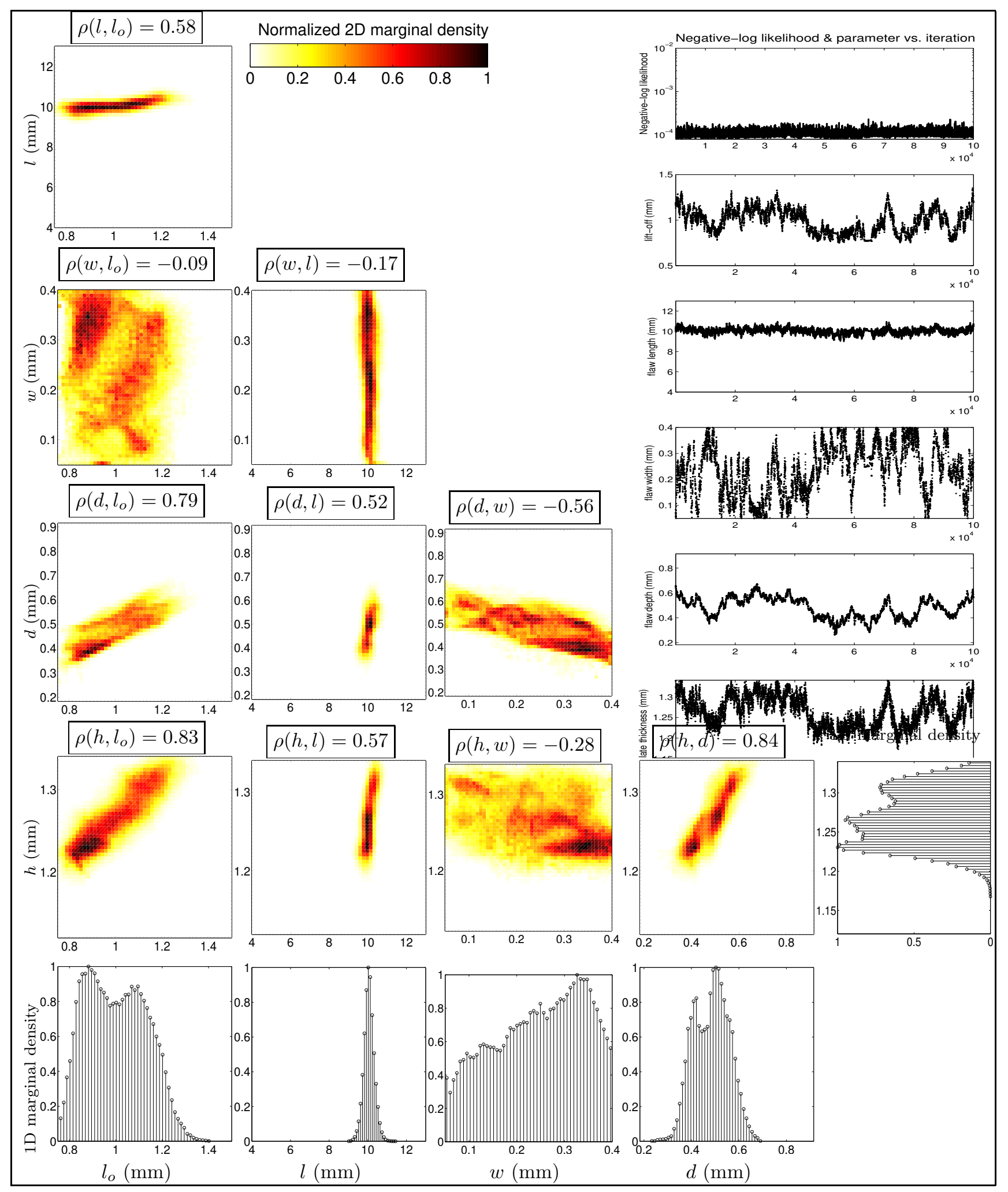

Figure 9: 2D and 1D marginal distributions estimated from histograms of MCMC samples for flaw $l 10 m m$, correlation coefficients are in boxed text at top of the 2D marginal posterior images and the corresponding negative-log likelihoods and parameter values versus MCMC iterations (on top-right). 


\section{A. Appendix: forward solver and metamodel generation}

\section{A.1. The employed forward solver}

In this work a Method of Moments (MoM) [28] based solver is employed (CIVA software [13]). Use of MoM in ECT signal modeling is well established, with origins in NdT by ECT about three decades ago [42] and before, with seminal works in geophysics [41, 46]. Considering canonical geometries, MoM does couple well with semi-analytical approaches for which a closed-form definition of the dyadic Green function is available in the literature [15]. The semi-analytical approach has been widely applied and validated on many test cases of interest in the NdT community [13] [42]. It enables to express a discontinuity within the inspected medium via a localized secondary source described with very few unknowns (only the discontinuities are meshed).

Thus, from reciprocity [4] one retrieves the effect of this secondary source on the primary one with a simple integration on the volume associated to the secondary source. E.g., in the ECT signal modeling of a planar multi-layered conductive non-ferromagnetic structure inspected with a coil in absolute mode, the reciprocity theorem reads as

$$
\begin{gathered}
\Delta Z=\frac{1}{I^{2}} \sum_{s=1}^{S} \int_{\Omega_{s}} \frac{1}{\sigma_{s}} \mathbf{J}_{s}^{i n c}(\mathbf{r}) \cdot \mathbf{p}_{s}(\mathbf{r}) d \mathbf{r}, \\
\mathbf{r} \in \Omega_{s}, \quad s \in 1, S .
\end{gathered}
$$

where $\Omega_{s}$ is the volume of the $s$-th flaw in the piece, $\mathbf{J}_{s}^{i n c}$ the current density induced by the coil in region $\Omega_{s}$ without flaw, $\mathbf{p}_{s}$ the electric dipole density associated to the flawed region $\Omega_{s}, I$ the coil driven current, and $\sigma_{s}$ the conductivity associated to the layer where the $s$-th dipole density is located. Quantities $\mathbf{p}_{s}$ are solutions of a system of $S$ integral equations. In the case of a Volume Integral Method (VIM) [42] they correspond to second-kind Fredholm equations

$$
\begin{gathered}
\mathbf{J}_{o}^{i n c}(\mathbf{r})=\mathbf{p}_{o}(\mathbf{r})-\sum_{s=1}^{S} f_{o}(\mathbf{r}) k_{o}^{2} \int_{\Omega_{s}} \underline{\mathbf{G}}_{o s}\left(\mathbf{r}, \mathbf{r}^{\prime}\right) \mathbf{p}_{s}\left(\mathbf{r}^{\prime}\right) d \mathbf{r}^{\prime}, \\
\mathbf{r}^{\prime} \in \Omega_{s}, \quad \mathbf{r} \in \Omega_{o}, \quad o \in[1, \ldots, S] .
\end{gathered}
$$

In the case of a Surface Integral Method (SIM) the set of state equations is obtained from first-kind Fredholm equation [9] [33] as

$$
\begin{aligned}
\mathbf{n}_{o} \cdot \mathbf{J}_{o}^{i n c}(\mathbf{r})=- & \sum_{s=1}^{S} f_{o}(\mathbf{r}) k_{o}^{2} \int_{\Omega_{s}} \mathrm{G}_{o s}^{n_{o} n_{s}}\left(\mathbf{r}, \mathbf{r}^{\prime}\right) \mathrm{p}_{s}\left(\mathbf{r}^{\prime}\right) d \mathbf{r}^{\prime}, \\
& \mathbf{r}^{\prime} \in \Omega_{s}, \quad \mathbf{r} \in \Omega_{o}, \quad o \in[1, \ldots, S] .
\end{aligned}
$$

where, for both equations (9) and (10), $\Omega_{s}$ is the source volume and $\Omega_{o}$ the observation one, in case of one flaw problem $\Omega_{s}=\Omega_{o}$. The system associated to (9) is made of $S$ vector integral equations, whereas for the system (10), the integral equations are reduced to scalar equations by projection onto the direction $\mathbf{n}_{o}$ of the flaw opening. In all these equations, the source point is at $\mathbf{r}^{\prime}=\left(x^{\prime}, y^{\prime}, z^{\prime}\right)$, while the observation point is at $\mathbf{r}=(x, y, z), \Omega_{s}$ representing the flaw domain. The contrast function $f_{o}(\mathbf{r})$, is $f_{o}(\mathbf{r})=\left[\sigma_{o}(\mathbf{r})-\sigma_{o}\right] / \sigma_{o}$ where $\sigma_{o}(\mathbf{r})$ and $\sigma_{o}$ are the flaw and the hosting layer conductivity, resp. The wave number is $k_{o}=\sqrt{i \omega \mu_{0} \sigma_{o}}$ and is associated to the layer hosting the $o$-th flaw. Subscripts $s$ and $o$ are employed to denote the quantities associated to the source and the observation, resp. If only one crack occupies the layered structure, then $s=o$. In (9) the electric-electric dyadic Green function $\underline{\mathbf{G}}_{o s}\left(\mathbf{r}, \mathbf{r}^{\prime}\right)$ is used to describe the electric field at point $\mathbf{r}$ due to a vector source located at $\mathbf{r}^{\prime}$. Its component $G_{o s}^{n_{o} n_{s}}\left(\mathbf{r}, \mathbf{r}^{\prime}\right)$ in (10) describes, when considering co-ordinate systems adapted to the source and observation flaws, the effects of a 
$\mathbf{n}_{s}$-oriented dipole placed at $\mathbf{r}^{\prime}$ onto the $\mathbf{n}_{o}$-oriented component of the electric field at observation point $\mathbf{r}$. To solve (9) and (10), flawed regions are uniformly meshed in parallelepiped cells, then MoM has been employed to build a solvable matrix linear system.

\section{A.2. Evaluation of metamodel accuracy of the experimental problem}

In this contribution, we employed metamodels to replace the standard forward solver within the MCMC-based optimization strategy. Its use should be justified in terms of accuracy in order to employ it as a "black-box". In this subsection we briefly discuss the accuracy of the metamodel and how it can be assessed.

First, we introduce some useful quantities that enable to evaluate the metamodel predictions. Since we deal with a functional output we have to define a suitable error metric for comparing forward solver outputs with the metamodel one. To this end, the Normalized L2 Error (NL2E) is defined as follows

$$
N L 2 E\left(\mathbf{x}_{i}\right)=\frac{\left\|\mathcal{F}\left\{\mathbf{x}_{i}\right\}-\mathcal{M}\left\{\mathbf{x}_{i}\right\}\right\|_{2}}{\left\|\mathcal{F}\left\{\mathbf{x}_{i}\right\}\right\|_{2}},
$$

where $\mathcal{F}\left\{\mathbf{x}_{i}\right\}$ stands for the forward solver evaluated on the $i$-th test set sample and $\mathcal{M}\left\{\mathbf{x}_{i}\right\}$ corresponds to the metamodel evaluated on the same sample. The Mean of NL2E (MNL2E) can thus be defined as

$$
M N L 2 E=\frac{1}{N_{\text {test }}} \sum_{i=1}^{N_{\text {test }}} N L 2 E\left(\mathbf{x}_{i}\right),
$$

where $N_{\text {test }}$ corresponds with the test set size.

In this appendix, we have chosen to assess the prediction performance (e.g., $M N L 2 E$ ) by employing a representative external test set having $N_{\text {test }}=500$ samples. The test samples have been generated by employing the CIVA software run on a set of input values generated through a Latin Hypercube Sampling (LHS) algorithm (Matlab function 1hsdesign). It is worthwhile to mention that MNL2E can be estimated also through cross-validation. To better underline the robustness of the developed metamodel, we have chosen to avoid cross-validation in our analysis since employed (with very similar results) in the database and metamodel generation phase.

In Fig. 10 we show the accuracy in prediction by comparing forward solver results with the metamodel one. A good agreement is found with low dispersion around the nominal gray line (i.e., the line representing the full agreement). Through the histogram plot shown in Fig. 10 we quantify the sample distribution versus the associated error $N L 2 E$ value. On the same plot the cumulative samples distribution curve in percentage is shown. This curve shows that about $64 \%$ of the test set samples has an error less than 6.06, which corresponds to the $M N L 2 E$. Moreover, we observe that about $85 \%$ of the test set samples has less or equal than $10 \%$ NL2 error. These results show that the discrepancy between metamodel and forward model results is fairly low. So, no particular bias is expected when the metamodel is employed instead of the "true" numerical solver.

Let us emphasize that in "real-life" experiment, due to conditions of inspection, imperfect matching between simulated and experimental data is always faced with, no matter the kind of metamodel or forward solver. So, the accuracy of the metamodel should be assessed by keeping into account simulations results and experimental data together. Unfortunately this kind of information can be established only a-posteriori upon availability of experimental data.

\section{A.3. Comments and remarks on the employed strategy}

In this work, the considered forward solver has been based on a validated semi-analytical approach which allowed to be, from the computational time point of view, almost independent with respect to the number of coil position considered. This choice is not mandatory. Accurate 


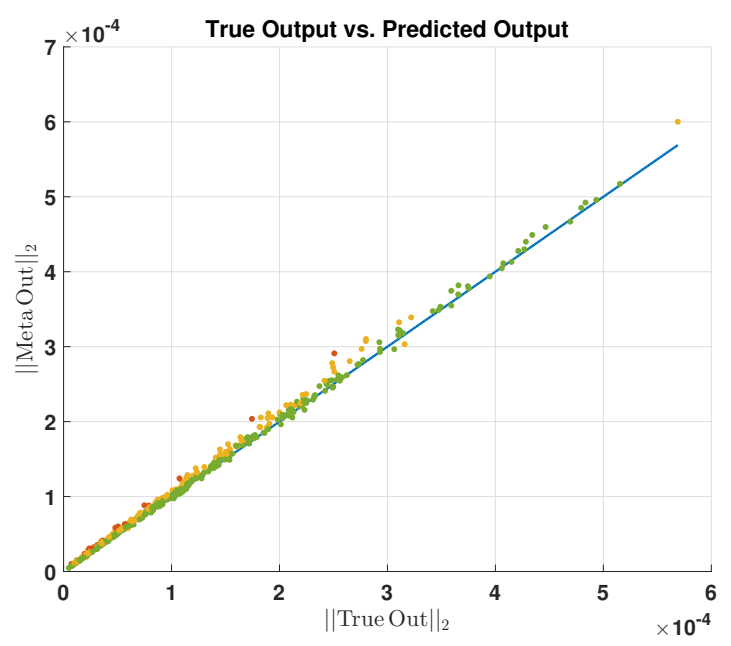

Figure 10: True versus predicted plot. Comparisons between the L2-norm of the forward solver outputs $\left(\|\right.$ True Out $\left.\|_{2}\right)$ and the metamodel predictions $\left(\|\right.$ Meta Out $\left.\|_{2}\right)$ based on a test set having 500 LHS samples.

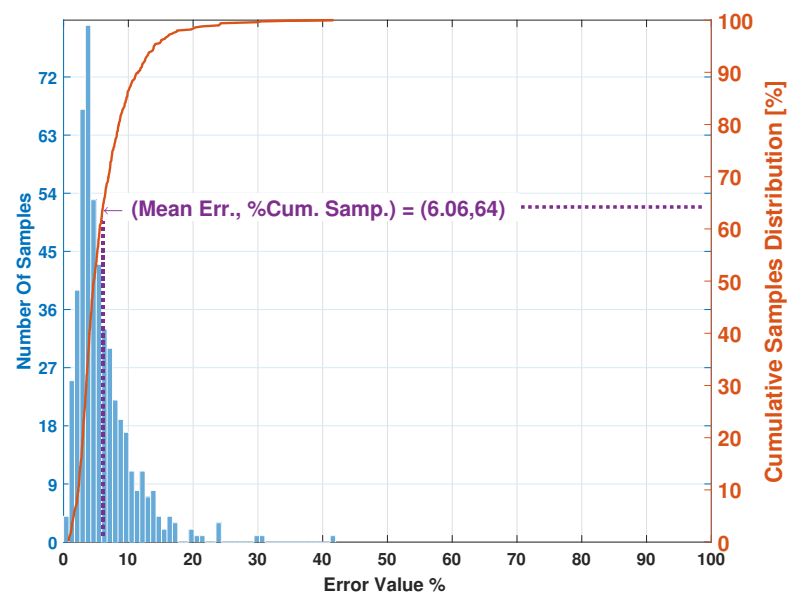

Figure 11: Errors frequency distribution histogram plot. The number of samples for a given error is shown on the ordinate left-hand axis. The solid line represents the cumulative curve associated to the percentage of samples (displayed on the right-hand side axis) that have an $N L 2$ error less or equal to the one displayed on the abscissa. 
numerical solvers could be used, e.g., Finite Element Method (FEM), yet their efficiency relies on the number of measurement points that one needs to simulate.

The analysis performed in this section in commonly carried out in order to build the suitable metamodel before employing it instead of the standard solver. That is, the metamodel is built and possibly tuned off-line. Once the quality of predictions is judged "good enough" for the targeted problem, the metamodel can be considered as proper replacement of the forward solver, i.e., as a black-box. So, the strategy to generate the suitable database may not be important if "only" the final prediction accuracy is targeted. In this context, the metamodel used in the on-line phase should not have an impact on the accuracy of the final inversion results. On the other hand, one could prefer a certain metamodel strategy if performance in off-line stage, i.e., the number of samples needed to achieve a certain accuracy level, and/or the on-line phase one in terms of interpolation time, i.e., the metamodel CPU time efficiency, should be optimized.

\section{Acknowledgement}

Support is from the French National Research Agency (ANR) project Bayesian Methods for the diagnosis and Probability of Detection assisted by Simulation (ByPASS).

\section{References}

[1] Abascal, J.F.P.J., Lambert, M., Lesselier, D., Dorn, O., 2008. 3-d eddy-current imaging of metal tubes by gradient-based, controlled evolution of level sets. IEEE Transactions on Magnetics 44, 4721-4729.

[2] Andrieu, C., Doucet, A., Holenstein, R., 2010. Particle Markov chain Monte Carlo methods. Journal of the Royal Statistical Society: Series B (Statistical Methodology) 72, 269-342.

[3] Andrieu, C., Thoms, J., 2008. A tutorial on adaptive MCMC. Statistics and Computing 18, 343-373.

[4] Auld, B.A., Jefferies, S.R., Moulder, J.C., 1988. Eddy-current signal analysis and inversion for semielliptical surface cracks. Journal of Nondestructive Evaluation 7, 79-94.

[5] Bilicz, S., 2013. Inversion of eddy-current testing signals using a fast interpolation over an optimal defect-database. IOS Press Volume 39: Electromagnetic Nondestructive Evaluation (XVII), 315-322.

[6] Bilicz, S., 2016. Sparse grid surrogate models for electromagnetic problems with many parameters. IEEE Transactions on Magnetics 52, 1-4.

[7] Bilicz, S., Lambert, M., Gyimóthy, S., 2010a. Kriging-based generation of optimal databases as forward and inverse surrogate models. Inverse Problems 26, 074012.

[8] Bilicz, S., Vazquez, E., Gyimothy, S., Pávó, J., Lambert, M., 2010b. Kriging for eddy-current testing problems. IEEE Transactions on Magnetics 46, 3165-3168.

[9] Bowler, J.R., 1994. Eddy current interaction with an ideal crack. I. The forward problem. Journal of Applied Physics 75, 8128-8137.

[10] Bowler, J.R., Norton, S.J., Harrison, D.J., 1994. Eddy current interaction with an ideal crack. II. The inverse problem. Journal of Applied Physics 75, 8138-8144. 
[11] Box, G.E.P., Tiao, G.C., 1992. Bayesian Inference in Statistical Analysis. John Wiley and Sons. Inc.

[12] Calderhead, B., 2014. A general construction for parallelizing Metropolis Hastings algorithms. Proceedings of the National Academy of Sciences 111, 17408-17413.

[13] CEA-LIST, 2014. CIVA : solutions pour les CND. Technical Report.

[14] Chen, Z., Aoto, K., Miya, K., 2000. Reconstruction of cracks with physical closure from signals of eddy current testing. IEEE Transactions on Magnetics 36, 1018-1022.

[15] Chew, W.C., 1999. Waves and Fields in Inhomogenous Media. Wiley-IEEE Press.

[16] Clauzon, T., Thollon, F., Nicolas, A., 1999. Flaws characterization with pulsed eddy currents NDT. IEEE Transactions on Magnetics 35, 1873-1876.

[17] Cotter, S.L., Roberts, G.O., Stuart, A.M., White, D., 2013. MCMC methods for functions: modifying old algorithms to make them faster. Statistical Science 28, 424-446.

[18] Cowles, M.K., Carlin, B.P., 1996. Markov chain Monte Carlo convergence diagnostics: a comparative review. Journal of the American Statistical Association 91, 883-904.

[19] De La Rosa, J.I., Fleury, G.A., Osuna, S.E., Davoust, M.E., 2006. Markov chain Monte Carlo posterior density approximation for a groove-dimensioning purpose. IEEE Transactions on Instrumentation and Measurement 55, 112-122.

[20] Doucet, A., de Freitas, N., Gordon, N., 2001. An Introduction to Sequential Monte Carlo Methods. Springer New York, New York, NY. pp. 3-14.

[21] Douvenot, R., Lambert, M., Lesselier, D., 2011. Adaptive metamodels for crack characterization in eddy-current testing. IEEE Transactions on Magnetics 47, 746-755.

[22] Forrester, A.I.J., Keane, A.J., 2009. Recent advances in surrogate-based optimization. Progress in Aerospace Sciences 45, 50-79.

[23] Girolami, M., Calderhead, B., 2011. Riemann manifold Langevin and Hamiltonian Monte Carlo methods. Journal of the Royal Statistical Society: Series B (Statistical Methodology) $73,123-214$.

[24] Green, P., 1995. Reversible jump MCMC computation and Bayesian model determination. Biometrika 82, 711-732.

[25] Green, P.J., Łatuszyński, K., Pereyra, M., Robert, C.P., 2015. Bayesian computation: a summary of the current state, and samples backwards and forwards. Statistics and Computing $25,835-862$.

[26] Haario, H., Laine, M., Mira, A., Saksman, E., 2006. DRAM: Efficient adaptive MCMC. Statistics and Computing 16, 339-54.

[27] Harney, H.L., 2003. Bayesian Inference: Parameter Estimation and Decisions. First ed., Springer.

[28] Harrington, R.F., 1993. Field Computation by Moment Methods. Wiley-IEEE Press. 
[29] Hastings, W.K., 1970. Monte Carlo sampling methods using markov chains and their applications. Biometrika 57, 97-109.

[30] Kleijnen, J.P.C., Beers, W.C.M.V., 2004. Application-driven sequential designs for simulation experiments: kriging metamodeling. The Journal of the Operational Research Society 15, 303-325.

[31] Metropolis, N., Rosenbluth, A.W., Rosenbluth, M.N., Teller, A.H., Teller, E., 1953. Equations of state calculations by fast computing machines. The Journal of Chemical Physics 21, 10871092.

[32] Miorelli, R., Reboud, C., Theodoulidis, T., Martinos, J., Poulakis, N., Lesselier, D., 2014. Coupled approach VIM-BEM for efficient modeling of ECT signal due to narrow cracks and volumetric flaws in planar layered media. NDT \& E International 62, 178-183.

[33] Miorelli, R., Reboud, C., Theodoulidis, T., Poulakis, N., Lesselier, D., 2013. Efficient modeling of ECT signals for realistic cracks in layered half-space. IEEE Transactions on Magnetics 49, 2886-2892.

[34] Mistral, Q., Charret, T., 2013. Array probe implementation (SMX) on EDF steam generator tubes, in: 10th International Conference on NDE in Relation to Structural Integrity for Nuclear and Pressurized Components, pp. 329-333.

[35] Neal, R.M., 2011. MCMC using Hamiltonian dynamics, in: Brooks, S., Gelman, A., Jones, G., Meng, X. (Eds.), Handbook of Markov chain Monte Carlo. Chapman \& Hall CRC Press. chapter 5, pp. 113-162.

[36] Netter, D., Leveque, J., Masson, P., Rezzoug, A., 2004. Monte Carlo method for transient eddy-current calculations. IEEE Transactions on Magnetics 40, 3450-3456.

[37] Norton, S.J., Bowler, J.R., 1993. Theory of eddy current inversion. Journal of Applied Physics $73,501-512$.

[38] Pakman, A., Paninski, L., 2014. Exact Hamiltonian Monte Carlo for truncated multivariate Gaussians. Journal of Computational and Graphical Statistics 23, 518-542.

[39] Pávó, J., Lesselier, D., 2006. Calculation of eddy current testing probe signal with global approximation. IEEE Transactions on Magnetics 42, 1419-1422.

[40] Queipo, N.V., Haftka, R.T., Shyy, W., Goel, T., Vaidyanathan, R., Tucker, P.K., 2005. Surrogate-based analysis and optimization. Progress in Aerospace Sciences 41, 1-28.

[41] Raiche, A.P., 1974. An integral equation approach to three-dimensional modelling. Geophysical Journal of the Royal Astronomical Society 36, 363-376.

[42] Sabbagh, J.B..L.S..H., 1990. Eddy-current probe impedance due to a surface slot in a conductor. IEEE Transactions on Magnetics 26, 889-892.

[43] Schmerr Jr., L.W., 2008. 2008 Eddy Current Benchmark Problems. Technical Report. World Federation of NDE Centers.

[44] Sekine, Y., Soyama, H., 2012. Using an eddy current method with inverse analysis to determine the thickness of the layer modified by cavitation peening at the surface of type $316 \mathrm{~L}$ austenitic stainless steel. NDT \& E International 46, 94-99. 
[45] Tierney, L., 1994. Markov chains for exploring posterior distributions. The Annals of Statistics $22,1701-1728$.

[46] Weidelt, P., 1975. Electromagnetic induction in three-dimensional structures. Journal of Geophysics 41, 85-109.

[47] Xie, S., Chen, Z., Takagi, T., Uchimoto, T., 2015. Quantitative non-destructive evaluation of wall thinning defect in double-layer pipe of nuclear power plants using pulsed ECT method. NDT \& E International 75, 87-95. 